\title{
Um microscópio caseiro simplificado
}

\author{
A simplified model of homemade microscope
}

\author{
Diogo Soga*1, Raul Dias Paiva Jr. $^{2}$, Michele Hidemi Ueno-Guimarães ${ }^{3}$, Mikiya Muramatsu ${ }^{1}$ \\ ${ }^{1}$ Instituto de Física da USP, Física Geral, Rua do Matão, 1371 prédio Ala 1, 05508-090, São Paulo, SP, Brazil \\ ${ }^{2}$ Centro Universitário Senac, São Paulo, SP, Brazil \\ ${ }^{3}$ Departamento de Física, Universidade Federal de Ouro Preto, Instituto de Ciencias Exatas e Biologicas, Campus Morro do \\ Cruzeiro, Ouro Preto, MG, Brazil
}

Recebido em 19 de Abril, 2017. Aceito em 14 de Maio, 2017.

\begin{abstract}
Neste trabalho é apresentada uma nova alternativa para a construção de microscópios caseiros, e uma nova forma de se obter lentes esféricas, utilizando-se de materiais mais baratos, o que facilita a construção e o uso do equipamento. A simplicidade da montagem e a facilidade do manuseio favorecem a aplicação do microscópio em locais com poucos recursos, além de torná-lo portátil. Além disso, são apresentadas sugestões de amostras a serem estudadas, pelos estudantes de diferentes disciplinas, o que pode fomentar a elaboração e a participação de alunos e professores em projetos interdisciplinares. O microscópio caseiro permite a visualização de pequenas estruturas, suas formas e cores, o que pode despertar o interesse do aluno pela análise de materiais e objetos, de uma forma muito mais instigante, que a mera observação a olho nu.
\end{abstract}

Palavras-chave: Óptica, lente esférica, microscópio caseiro, instrumentação.

This work presents a project for a homemade microscope with a new and alternative way to get spherical lenses, using cheap materials, which can facilitates its construction and use. The simplicity in its assembling and handling encourages its use in poor resourced places and additionally makes it a very portable instrument. Furthermore, suggestions of samples to be examined by students of different disciplines are also presented. This may encourage students and teachers to be more involved in elaboration of and/or participation in interdisciplinary projects. This homemade microscope enables visualization of small structures, their shapes and colors. The use of this simple instrument can grow in the students the desire and interest in the study and analysis of materials and objects in a much more instigating way, then by simple unaided eye observation.

Keywords: Optics, spherical lens, homemade microscope, instrumentation.

\section{Introdução}

Nas aulas de Óptica são estudados os fenômenos de refração, reflexão, difração etc. O estudo das lentes é um exemplo da aplicação do fenômeno da refração, no qual são estudadas a formação de imagens e o cálculo dos parâmetros do sistema óptico, como o comprimento focal e o aumento.

As lentes são objetos importantes no dia-a-dia, elas são empregadas em óculos para correção de defeitos visuais, equipamentos eletrônicos com diodos laser, impressoras laser etc. Outros equipamentos também contêm lentes, como os telescópios ópticos dos observatórios espaciais, que possibilitam visualizar os planetas, as estrelas e as galáxias, sem a necessidade de sair do planeta Terra. Por outro lado, as lentes também possibilitam ir do mundo macro ao mundo micro, por meio dos microscópios ópticos compostos [1-3], nos quais observa-se partículas, tecidos, líquidos, cristais etc.

O microscópio óptico, também chamado de microscópio óptico composto, é um instrumento utilizado pelas Ciên-

*Endereço de correspondência: diogosp@usp.br cias Básicas, tais como, a Física, a Química e a Biologia, para examinar objetos pequenos; o tamanho deles pode variar de alguns milímetros até micrômetros. E ele é utilizado também pela Medicina, Engenharia, Geologia, perícia criminal da Polícia etc.

A utilização de microscópios ópticos compostos em aulas esbarra em algumas dificuldades. Um microscópio didático comercial não é tão barato e o seu uso ainda requer energia elétrica, manutenção, amostras, lâminas de vidro etc. Nem todas as escolas dispõem de tais equipamentos, condições de trabalho e local adequado. A aplicação de um microscópio caseiro vem suprir essa possível lacuna. Tanto o seu uso quanto a sua construção têm o carácter de atividade didática. E tal atividade pode ser caracterizada como uma instrumentalização do Ensino.

A instrumentalização [4] na área de Ensino auxilia o educador a trazer a realidade mais perto do aluno, com a visualização dos fenômenos físicos e de suas aplicações. Segundo o trabalho de Araújo e Abib [5], que analisou diversos trabalhos publicados em três publicações nacionais (no período de 1992 a 2001), verificou-se que os 
autores são unânimes em defender o uso de atividades experimentais em sala de aula, e há dois aspectos que atestam a eficiência desta estratégia: a capacidade de estimular a participação do aluno; e propiciar um ambiente motivador. Vejamos dois exemplos: Silva e Muramatsu [6] apresentam um aparato simples para medir o índice de refração da água, sem a necessidade de um refratômetro; enquanto que Rocha e outros [7] apresentam uma demonstração, que visa explicar o céu azul e o vermelho do pôr-do-sol, utilizando um aquário com água e leite em pó.

Este trabalho propõe a construção de um microscópio caseiro, a partir de materiais que podem ser encontrados dentro de casa, de baixo custo, e de aplicações simples. É possível transportar esse microscópio caseiro, a diversos locais, pois ele é leve e de reparação simples. Tratase de uma lente esférica de vidro presa a um suporte de papelão. Apesar de sua simplicidade, ele apresenta qualidades. Com este modelo é possível estudar a amostra em quase toda a extensão, ao contrário do imposto por alguns modelos de microscópios caseiros.

A obtenção da lente é barata, sendo uma esfera de vidro de diâmetro da ordem de milímetros, e com um aumento grande, da ordem de uma centena de vezes.

\section{Microscópio Óptico}

O microscópio óptico composto [1 3] possui duas lentes, a objetiva e a ocular, para formar uma imagem ampliada da amostra sob análise. A lente objetiva forma e amplia a imagem inicial. Depois, esta imagem é projetada para o olho do observador pela lente ocular, com mais um aumento.

O microscópio óptico simples é um aparelho que possui apenas a lente objetiva. Esse modelo de microscópio pode ser confundido com uma lupa, mas é semelhante ao microscópio de Antony van Leeuwenhoek 8]. Tal microscópio é composto por uma lente esférica, presa a um suporte, com alguns mecanismos de posicionamento da lente em relação à amostra. Leeuwenhoek apresentou muitos trabalhos na área da Biologia com esse equipamento (estudos sobre: sementes, ossos, pele, escamas de peixe, cascas de ostras, língua, nervos, fibras musculares, sistema circulatório de peixes, olhos de insetos, vermes parasitas, fisiologia de aranha etc.).

Também temos que considerar que a lupa [1] é composta por uma lente biconvexa, de diâmetro da ordem de $10 \mathrm{~cm}$ e comprimento focal também da ordem de centímetros, com aumentos de 4 a 10 vezes. Pórem, as lentes usadas em microscópios têm diâmetros de alguns milímetros e aumentos muito maiores, o que corresponde ao modelo proposto. Dessa maneira, o aparato apresentado poderia ser chamado de microscópio simples ao invés de lupa, mas será denominado microscópio caseiro.

$\mathrm{Na}$ Internet é possível encontrar alguns trabalhos que descrevem a construção de microscópios caseiros 9 [13], visando estimular o Ensino de Ciências em sala de aula. O modelo proposto pelo trabalho de Vannoni e outros 12 apresenta um formato que assemelha-se ao microscópio composto, apresentado em alguns livros didáticos [1] 3 . Tal formato pode ser composto por duas lentes (lentes objetiva e ocular) ou três lentes (lentes objetiva, de campo e ocular), instalados em um tubo junto a um suporte vertical. Mas em formato diferente, o trabalho de Sepel e outros [9] apresenta um modelo de microscópio construído com um gargalo de garrafa plástica (PET), onde a rosca do gargalo funciona como um sistema para ajuste de foco da lente, que está fixada na tampa. O modelo apresentado no trabalho de Myint e outros 13 difere dos demais porque utiliza uma gota de água para atuar como uma lente objetiva, e o suporte dessa lente também tem formato diferenciado. Estes projetos necessitam de muito material e de ferramentas, que nem sempre são facilmente acessíveis, alguns deles possuem características físicas que não permitem a total exploração da amostra de maneira fácil, ou ainda envolvem o desmonte de equipamentos de informática para obter a lente.

Simplificar o aparato de demonstração reduz os custos de produção, e facilita que o aluno veja a Física envolvida, pois não há elementos que desviem a sua atenção. $\mathrm{O}$ modelo proposto neste trabalho consiste de uma lente esférica e um suporte de papelão. A lente esférica é a peça principal na construção do microscópio caseiro. A sua obtenção pode ser simples como descrita na próxima seção.

\section{Materiais e Métodos}

A seguir descrevemos a obtenção da lente esférica de vidro e a construção do microscópio caseiro.

\subsection{Lente Esférica}

Uma esfera de vidro transparente pode ser encontrada em algumas válvulas de recipientes de géis ou loções. Esta esfera pode funcionar como uma lente esférica para o microscópio caseiro. Um exemplo de recipiente pode ser visualizado na figura 1(a), que é uma embalagem de sabonete líquido, e a válvula da embalagem na figura 1(b). A lente esférica está dentro do mecanismo de aplicação do fluido (figura 1(c)). A seta indica a lente esférica, próxima da posição na válvula. Na figura $1(\mathrm{~d})$ vê-se a lente esférica de vidro. A lente utilizada neste trabalho foi retirada de um recipiente de sabonete líquido comercial, que iria para descarte após o seu consumo. A esfera tem diâmetro aproximado de $4 \mathrm{~mm}$.

As esferas de vidro podem ser encontradas em:

- embalagens de sabonete líquido de marcas diferentes;

- embalagem plástica com pulverizador de líquidos (figura 2); geralmente a esfera de vidro encontra-se na parte superior da válvula, na região indicada pela circunferência preta da figura 2(c); 


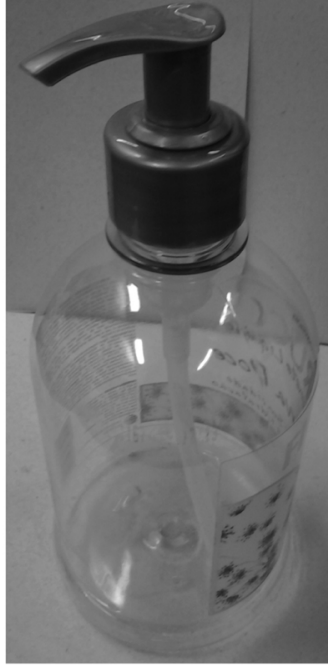

(a)A embalagem vazia.

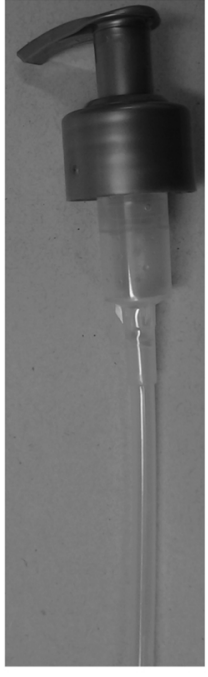

(b)A válvula da embalagem.

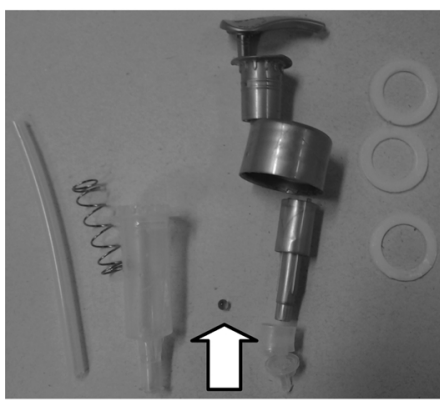

(c)As peças da válvula desmontadas e a seta branca indica a lente esférica de vidro.

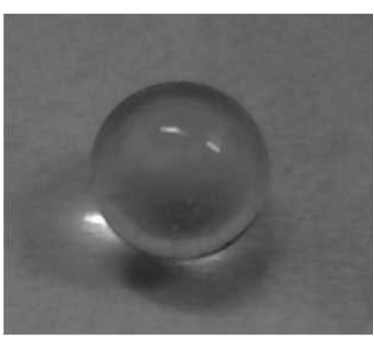

(d)A lente esférica de vidro.
Figura 1: Recipiente de sabonete líquido.

- embalagem de líquido ou creme avulsa com válvula (figura 3); geralmente a esfera de vidro (figura 3(b)) encontra-se junto à mola.

A embalagem plástica, quando for transparente e flexível, pode ser usada para substituir as lâminas de vidro, usadas para suportar as amostras, que serão analisadas em sala de aula com o microscópio caseiro. A utilização da embalagem plástica reduz ainda mais o custo de fabricação das lâminas de amostras.

A lente esférica (ou ball lens) utilizada neste trabalho tem dimensões, tais que a sua espessura não pode ser desprezada, como é feito para calcular o comprimento focal (f) da lente por meio da fórmula do fabricante [2]. Uma dedução formal do comprimento focal de uma lente esférica pode ser encontrada no trabalho de Soga e outros 14. Seja $R$ o raio da lente esférica e $n_{2}$ o índice de refração do material da lente, o comprimento focal $f$ é dado por:

$$
f=\frac{R n_{2}}{\left[2\left(n_{2}-1\right)\right]}
$$

Para uma lente de vidro $\left(n_{2}=1,5\right)$ e diâmetro de 4 $\mathrm{mm}(R=2 \mathrm{~mm})$, o comprimento focal é $f=3,0 \mathrm{~mm}$.

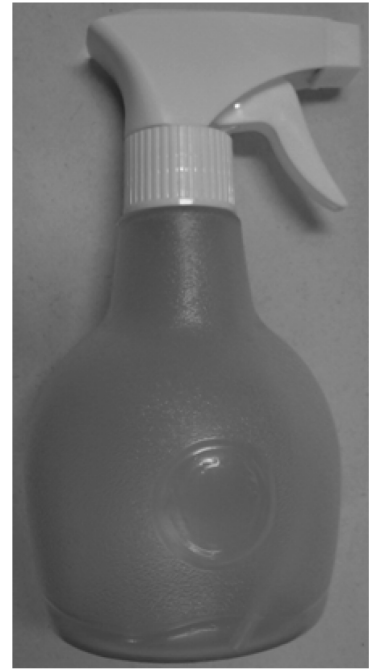

(a)A embalagem montada.

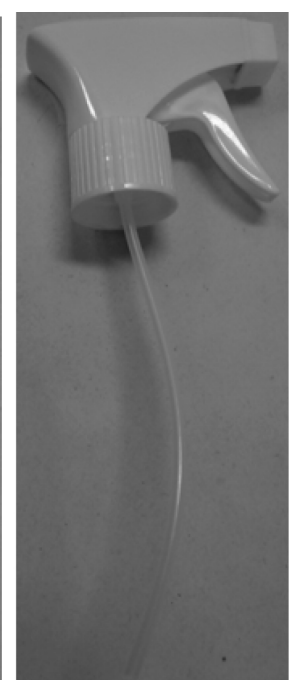

(b)A válvula da embalagem.

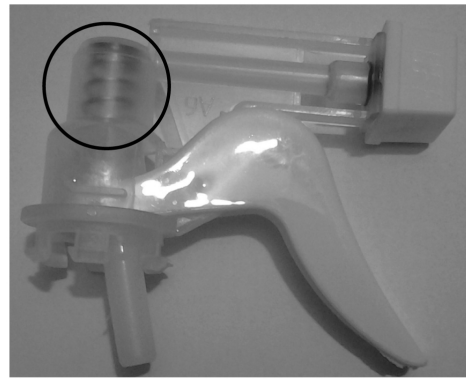

(c)A parte superior da válvula sem a capa.

Figura 2: Embalagem de pulverizador de líquidos.

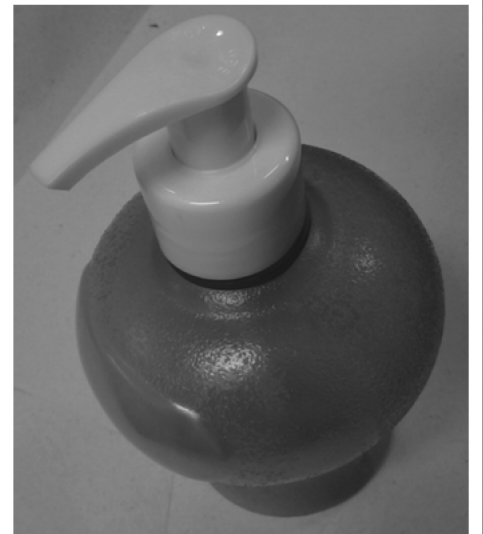

(a)A embalagem montada.

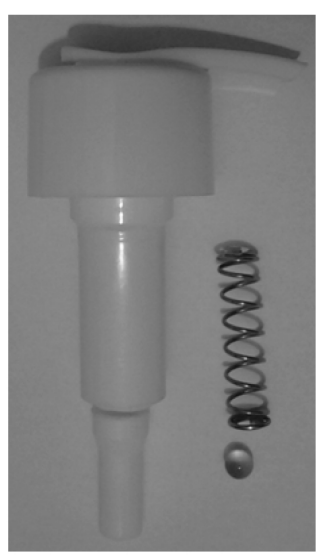

(b)A válvula da embalagem.
Figura 3: Recipiente de sabonete líquido, vendido avulso.

Subtraindo o raio da lente, a distância da superfície da lente até a amostra é de $1 \mathrm{~mm}$.

O aumento ( $M$, magnification) da imagem para este caso, depende da capacidade visual do olho humano 15 e é dado pela expressão:

$$
M=\frac{250}{f},
$$


onde 250 é a distância mínima, em milímetros, a partir do olho, para o qual é possível obter uma imagem distinta, e $f$ é o comprimento focal da lente, em milímetros. Calculando para uma lente de diâmetro $4 \mathrm{~mm}$, tem-se que o valor do aumento dessa lente é $M \sim 88 \mathrm{X}$, que é um aumento considerado grande. Com esta lente é possível visualizar objetos pequenos.

\subsection{Construção do microscópio caseiro}

O microscópio caseiro é constituído por uma lente esférica em um suporte de papelão. Tomamos um pedaço de papelão (figura $4(\mathrm{a})$ ), $30 \times 120 \mathrm{~mm}^{2}$, que foi dobrado ao meio, então foram feitos dois orifícios alinhados, de forma quadrada, de dimensões inferiores ao diâmetro da lente esférica. Sendo a lente utilizada de diâmetro de 4 $\mathrm{mm}$, então os orifícios quadrados têm as dimensões de 3 x $3 \mathrm{~mm}^{2}$, na foto a lente esférica está sobre o orifício de baixo. Ao dobrar o papelão, a lente é presa entre eles e acomodada nos orifícios, as bordas do papelão são presas com fita adesiva. É preciso colocar um grampo, próximo a lente, para que ela não saia de sua posição (figura 4(b)). Parte do papelão utilizado provém de embalagens de produtos que seriam descartados. Durante o uso desses microscópios, não há contato dos dedos com a lente, o que evita que ela se suje, além de facilitar a manipulação da peça.

O uso do microscópio caseiro é fácil. Basta segurar o microscópio perto da amostra, de modo que o observador olhe através da lente esférica e varie a distância da lente à amostra, até obter uma imagem focalizada. O posicionamento da amostra é feito diretamente pela mão do observador e deve-se alinhar o conjunto olholente-amostra. Aproxima-se o olho da lente, até que a imagem possa ser vista. Varia-se a distância lente-objeto até que a imagem fique nítida. Essa distância é da ordem de milímetros, como foi visto na subseção 3.1 .

A fonte de luz é a própria luz do ambiente. Caso a amostra seja muito escura, segure-a, juntamente com o microscópio caseiro e direcione-os a uma janela ou a uma parede bem iluminada. Evite direcionar o conjunto ao Sol ou para uma lâmpada, pois a lente pode concentrar os raios de luz, o que pode causar danos ao olho.

Para auxiliar o manuseio (vide figura 5), sugere-se prender a amostra (A) ao microscópio caseiro, com um

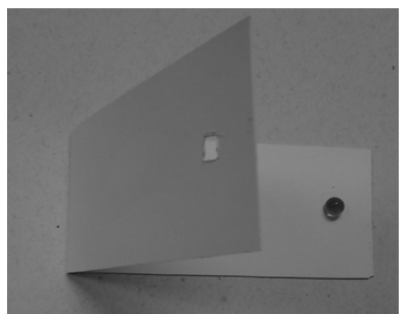

(a)O suporte com os dois orifícios e a lente esférica.

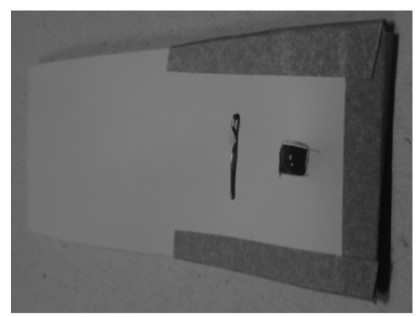

(b)O microscópio caseiro montado.
Figura 4: O microscópio caseiro.

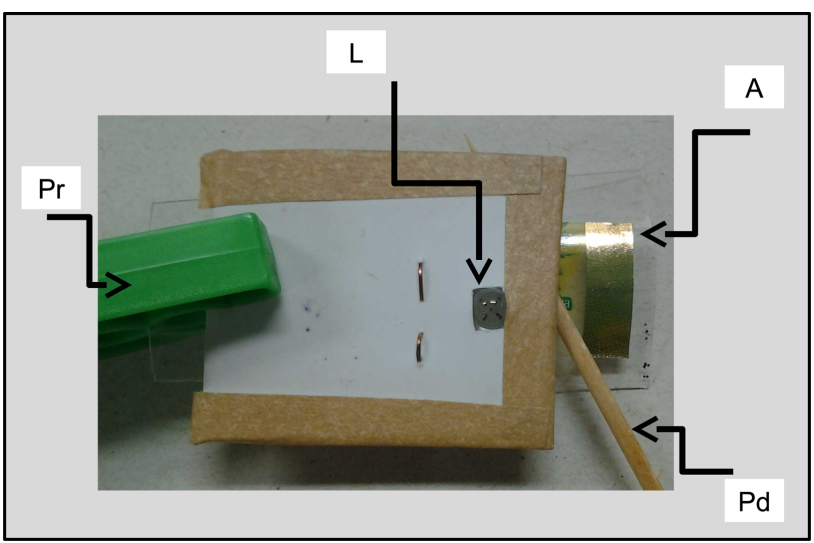

Figura 5: $O$ aparato na configuração de sugestão de uso: $(A)$ amostra, $(\mathrm{Pr})$ prendedor de roupa, $(\mathrm{Pd})$ palito de dente e $(\mathrm{L})$ lente esférica.

prendedor de roupa $(\mathrm{Pr})$, de maneira a aproximar a lente do microscópio da amostra. A distância entre a amosta e o microscópio pode ser aumentada ou diminuída, utilizando-se um palito de dente $(\mathrm{Pd})$. Devido a sua forma cônica na ponta, ele pode ser mais ou menos introduzido, dependendo da distância que se necessita, entre a entre a amostra e o microscópio. Olhe através da lente (L) para observar a imagem.

\subsection{Amostras Utilizadas}

Neste trabalho foram utilizadas cinco amostras: a) duas eram lâminas de microscopia comerciais de objetos padrões (perna de inseto e folha de pinheiro); b) e três foram preparadas pelos autores (rótulo impresso, papel, fibra de corda).

As amostras comerciais foram preparadas para o treinamento de estudantes, utilizando microscópios ópticos compostos. Elas são seguras, pois foram lacradas entre duas lâminas de vidro e preparadas quimicamente, para não se deteriorem com o tempo.

As amostras preparadas pelos autores são simples, de fácil acesso e seguras. Usando lâminas de plástico transparentes, de embalagens descartadas, alguns objetos foram fixados nas lâminas, por fita adesiva transparente de material plástico.

\subsection{Cuidados}

A abordagem deste trabalho inclui a reciclagem, visando facilitar o acesso aos materiais, para a construção de um microscópio caseiro. Entretanto, alguns cuidados devem ser tomados:

- não reaproveitar embalagens e materiais de produtos químicos e biológicos, utilizar preferencialmente embalagens de alimentos ou de cosméticos simples e inócuos, como sabonetes líquidos, cremes e loções para mãos; ainda assim, realizar uma lavagem cuidadosa, para remover qualquer resto do produto, evitando acidentes; 
- não tentar abrir embalagens metálicas tipo spray, pois elas contêm material pressurizado e possivelmente inflamável;

- não olhar através da lente em direção ao sol, à luz laser, nem a outras fontes de luz de grande intensidade;

- não deixar que fragmentos da amostra caiam nos olhos.

\section{Resultados}

Nesta seção apresentamos as fotos das amostras estudadas e as imagens dessas amostras obtidas com o microscópio caseiro. As imagens são coloridas, o que contribui positivamente para a visualização de detalhes das amostras.

Na figura 6, tem-se a perna de um inseto (amostra comercial). Na imagem (figura 6(b)) é possível ver os pelos da perna e parte da estrutura interna do inseto. Vêse que, no centro da imagem ela é nítida, com contornos bem definidos e bom contraste. Porém, nas bordas da imagem, não se tem nitidez e não é possível identificar detalhes da amostra. Esses erros de formação de imagem são chamados de aberrações da lente [2]. As melhores lentes usam vidros mais caros, formatos específicos e são agrupadas (duas ou mais lentes coladas). Para os propósitos deste trabalho, essas aberrações são aceitáveis.

Por meio da imagem do microscópio caseiro, é possível visualizar que a perna não é totalmente opaca, bem como parte da sua estrutura, como os pelos que cobrem algumas regiões.

A amostra da figura 7 (a) é um pedaço de uma folha de pinheiro (amostra comercial), a amostra tem comprimento de 1,2 mm. A olho nu não é possível visualizar muitos detalhes da amostra. Na imagem do microscópio caseiro (figura 7(b)) veem-se detalhes da estrutura da amostra, além disso, é possível ver os formatos, tamanhos e cores diferentes de cada região da estrutura da amostra.

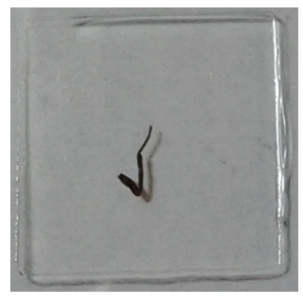

(a)A amostra em lâmina, o quadrado mede $12 \times 12 \mathrm{~mm}^{2}$

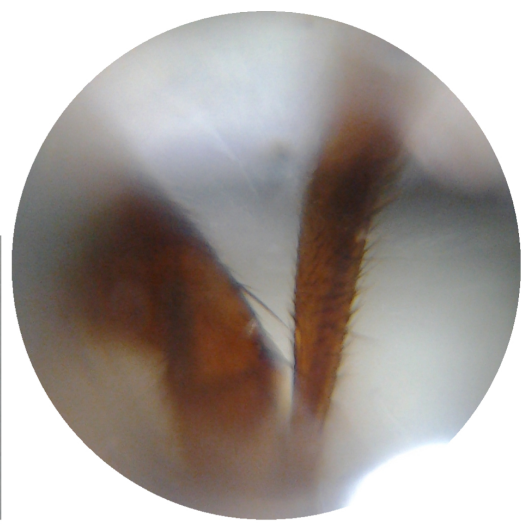

(b)Imagem obtida com o microscópio caseiro.

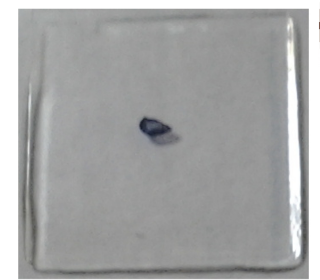

(a)A amostra em lâmina, o quadrado mede $12 \times 12 \mathrm{~mm}^{2}$.

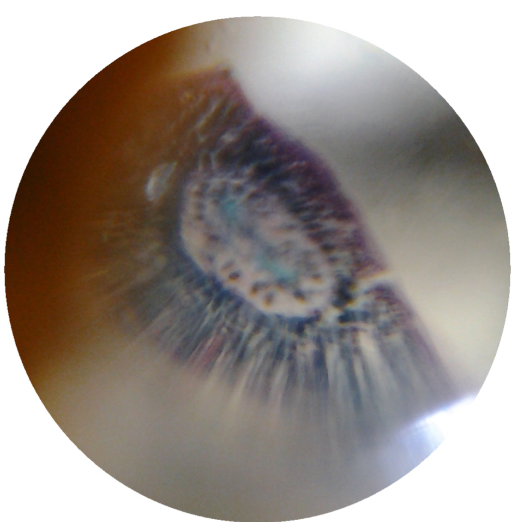

(b)Imagem da amostra.
Figura 7: Amostra de folha de pinheiro.

Na figura 8(a), têm-se dois pedaços de um rótulo de plástico impresso, nos quais há desenhos e letras coloridas. Na figura 8(b), vê-se que a região dourada é formada por várias cores e pontos impressos, ao invés de apenas pontos dourados. Na figura 8(c) vê-se que a região branca contém pontos pretos que não são visíveis a olho nu sobre um fundo de cor branca. Dessa forma, pode-se notar que a figura impressa é formada por pontos, e as cores vistas a olho nu são combinações de outras cores, até mesmo no caso da cor branca.

Na figura 9(a), tem-se um pedaço de papel verde, cuja borda direita foi rasgada. Na figura $9(\mathrm{~b})$ vê-se que a cor verde não é a predominante e sim, que é um papel amarelo, com pontos nas cores verde e preta. Além disso,

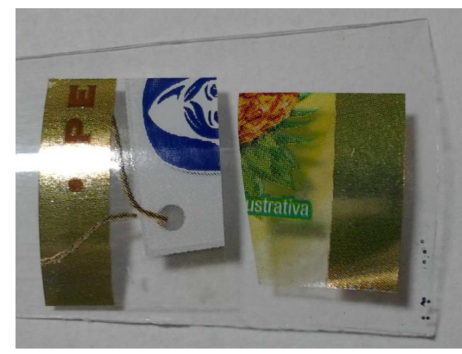

(a)A amostra em lâmina, que mede $30 \times 20 \mathrm{~mm}^{2}$

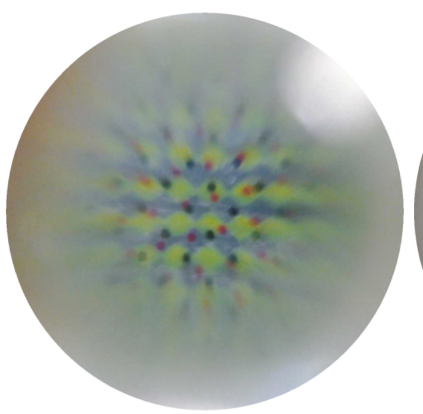

(b)Imagem da amostra da região dourada.

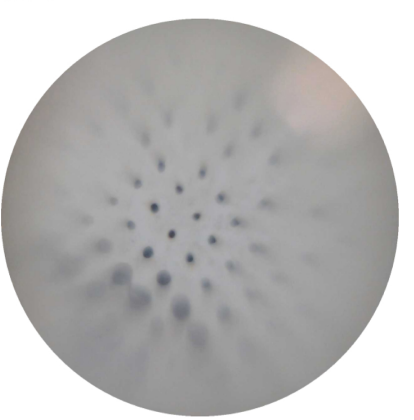

(c)Imagem da amostra da região branca.
Figura 8: Amostra de um rótulo impresso.

Figura 6: Amostra de perna de inseto. 


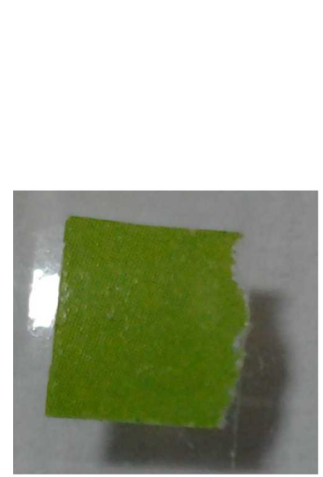

(a) A amostra em lâmina, o retângulo mede $8 \times 7 \mathrm{~mm}^{2}$.

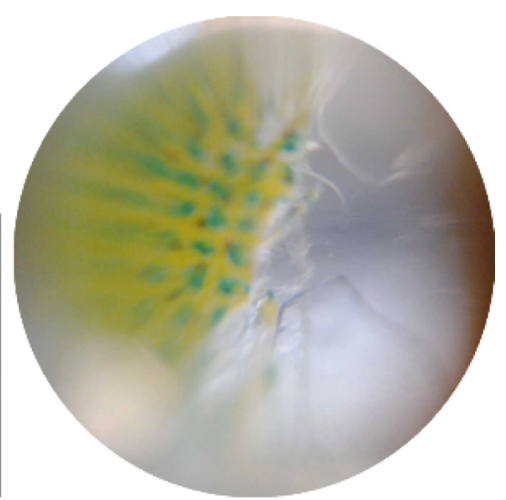

(b)Imagem da amostra da borda rasgada.
Figura 9: Amostra de papel impresso e rasgado na borda direita.

percebem-se na borda rasgada as fibras que formam o papel.

Na figura 10(a), tem-se um pedaço de corda de fibras. Na figura 10(b) vê-se que cada fio da corda é formado por vários fios, bem mais finos, e que a borda reta é o ponto onde ela foi cortada.

As amostras estudadas acima são muito diferentes entre si, o que indica a potencialidade de uso do microscopio proposto para diversas disciplinas.

A maioria dos materiais utilizados foram reaproveitados de materiais que seriam descartados. $\mathrm{E}$ a simplicidade do modelo proposto garante a aplicabilidade em sala de aula ou como atividade lúdica para o incentivo do Ensino de Ciências.

\section{Conclusão}

Neste trabalho foi descrita a construção de um microscópio caseiro simplificado, a partir de materiais de fácil acesso, utilizando materiais recicláveis, e de baixo custo. A simplicidade da montagem e a facilidade do manuseio favo-

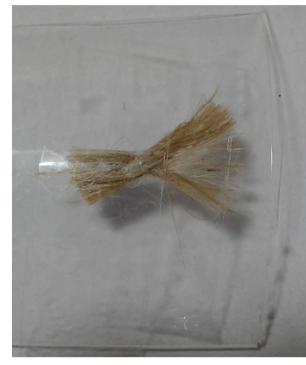

(a)A amostra em lâmina, o quadrado mede $10 \times 15 \mathrm{~mm}^{2}$

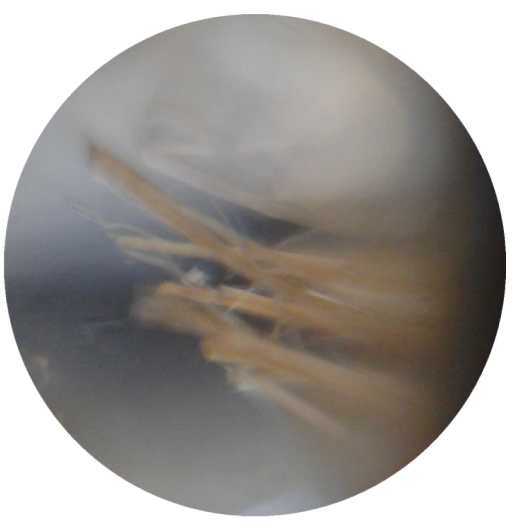

(b)Imagem da amostra.
Figura 10: Amostra de corda de fibras. recem a aplicação do microscópio em locais com poucos recursos, além de torná-lo portátil. Esse instrumento permite ao professor e ao estudante acessarem o mundo microscópico, sem aparelhos complexos ou investimento de grandes recursos, incentivando os alunos ao estudo de Ciências. Um aspecto, importante a ressaltar, é a possibilidade de ele ser utilizado em eventos, como por exemplo, Feira de Ciências.

Apesar de algumas distorções nas imagens, fica demonstrado que esse microscópio caseiro simplificado permite a visualização de pequenas estruturas, suas formas e cores, o que pode despertar o interesse do aluno pela análise de materiais e objetos, de uma forma muito mais instigante, que a mera observação a olho nu. Para a maioria dos estudantes, os objetos são como eles conseguem visualizá-los. Eles não imaginam a complexa rede de que são formados e os detalhes que compõem suas estruturas.

Além disso, seria possível trabalhar em conjunto com outras disciplinas, pois como foi visto, não só a Física permite um olhar mais detalhado do seu mundo, mas também a Biologia, a Química e a natureza de maneira geral.

\section{Agradecimentos}

Os autores agradecem o suporte técnico de Claudio Hiroyuki Furukawa (Instituto de Física da USP) e Simone Perche de Toledo (Instituto de Física da USP).

\section{Apêndice}

\section{Custos de materiais}

Abaixo são apresentados os valores de alguns itens mencionados no trabalho e a época da tomada de preços, na cidade de São Paulo (SP):

- microscópio óptico composto, $\mathrm{R} \$ 1.000,00$, abril de 2014

- embalagem plástica com pulverizador de líquidos, $\mathrm{R} \$ 3,00$, abril de 2014;

- embalagens de líquidos e cremes avulsas com válvula, $\mathrm{R} \$ 3,00$, abril de 2014 .

\section{Referências}

[1] D. Halliday, R. Resnick e K.S. Krane, Física - Volume 4 (LTC, São Paulo, 2014), $5^{\mathrm{a}}$ ed.

[2] E. Hecht, Óptica (Fundação Calouste Gulbenkian, Lisboa, 1991).

[3] J.D. Cutnell, Física v.2 / John d. Cutnell, Kenneth W. Johnson (Editora LTC, Rio de Janeiro, 2006).

[4] W.L. Almeida, F.M.M. Luz, J.B. Silva, S.L.R. Silva e A.M. Brinatti, Caderno Brasileiro Ensino de Física 30, 396 (2013).

[5] M.S.T. Araújo e M.L.V.S. Abib, Revista Brasileira de Ensino de Física 5, 176 (2003).

[6] F.M. Silva e M. Muramatsu, Física na Escola 8, 46 (2007). 
[7] M.N. Rocha, T.G. Fujimoto, R.S. Azevedo e M. Muramatsu, Revista Brasileira de Ensino de Física 32, 3501 (2010).

[8] A.J.M. Wollman, R. Nudd, E.G. Hedlund e M.C. Leake, Open Biology 5, 150019 (2016).

[9] L.M.N. Sepel, J.B.T. Rocha e E.L.S. Loreto, Genética na Escola 6, 1 (2011).

[10] WAO Corporation Kabushikigaisha, Let's Make Microscope - WAO, disponível em http://www. youtube.com/ watch?v=UTADdH3Q_sQ, acesso em 23/04/2014.

[11] PIBID UFSM CAPES, Construindo Microscópios Reciclados, disponível em http://www . youtube.com/watch? v=7-PwVOkfaKM acesso em 23/04/2014.

[12] M. Vannoni, P.K. Buah-Bassuah e G. Molesini, Physics Education 42, 385 (2007).

[13] H.H. Myint, A.M. Marpaung, H. Kurniawan, H. Hattori e K. Kagawa, Physics Education 36, 97 (2001).

[14] D. Soga, R.D. Paiva Jr. e M. Muramatsu, Revista Brasileira de Ensino de Física 39, 3506 (2017).

[15] F.A. Jenkins e H.E. White, Optical Instruments, Fundamentals of Optics (McGraw-Hill, Singapura, 1981), $4^{\mathrm{a}}$ ed. 